\title{
Dynamics of quasiparticle trapping in Andreev levels
}

\author{
D. G. Olivares and A. Levy Yeyati* \\ Departamento de Física Teórica de la Materia Condensada, Condensed Matter Physics Center (IFIMAC), \\ and Insitituto Nicolás Cabrera, Universidad Autónoma de Madrid, E-28049 Madrid, Spain \\ L. Bretheau, Ç. Ö. Girit, H. Pothier, and C. Urbina \\ Quantronics Group, Service de Physique de l'Etat Condensé (CNRS, URA 2464), IRAMIS, CEA-Saclay, 91191 Gif-sur-Yvette, France
}

(Received 26 September 2013; published 4 March 2014)

\begin{abstract}
We present a theory describing the trapping of a quasiparticle in a prototypical Josephson junction, a singlechannel superconducting weak link. We calculate the trapping and untrapping rates associated to absorption and emission of both photons and phonons. We show that the presence of an electromagnetic mode with frequency smaller than the gap gives rise to a rather abrupt transition between a fast-relaxation regime dominated by coupling to photons and a slow-relaxation regime dominated by coupling to phonons. This conclusion is illustrated by the analysis of a recent experiment [Zgirski, Bretheau, Le Masne, Pothier, Esteve, and Urbina, Phys. Rev. Lett. 106, 257003 (2011)] measuring the dynamics of quasiparticle trapping in a superconducting atomic contact coupled to a Josephson junction. With realistic parameters, the theory provides a semiquantitative description of the experimental results.
\end{abstract}

DOI: 10.1103/PhysRevB.89.104504

\section{INTRODUCTION}

There are several external mechanisms that undermine the quantum coherence of superconducting circuits being explored for quantum information processing [1]. Their influence has been reduced over the years by new designs that minimize the coupling with external degrees of freedom. However, a fundamental intrinsic decoherence process arises from the coupling of the qubit variables to superconducting quasiparticles tunneling through the Josephson junctions of the circuits. Although, in principle, the superconducting gap $\Delta$ provides an inherent protection against low-energy excitations at low temperatures, in practice, there are residual nonequilibrium quasiparticles that can rule the behavior of the circuits [2-8]. As shown in a recent experiment [9], this is particularly true for weak links containing channels of high transmission, where localized excitations occupying Andreev levels of energy below $\Delta$ become possible. This has important consequences for the corresponding proposed qubits designs [10-13]. Furthermore, single quasiparticle trapping in localized levels could be detrimental in experiments proposed to detect "Majorana bound states" in condensed matter systems since their topological protection relies on parity conservation [14]. Understanding the dynamics of relaxation of quasiparticles in superconducting weak links is therefore an important present-day issue.

We report here on a theory highlighting the role of the electromagnetic environment in this dynamics. In experiments, Josephson junctions or weak links are very often embedded in electrical circuits having electromagnetic modes at frequencies lower than the superconducting gap. The environment can be a resonator intentionally coupled to the junction like in Ref. [9], or the plasma mode of another junction placed in parallel like in Ref. [15]. We show that if the mode impedance is large enough, it rules the quasiparticle dynamics when the sum of the Andreev level energy and of the energy of the mode exceed the superconducting gap.

\footnotetext{
*Corresponding author: a.l.yeyati@uam.es
}

PACS number(s): 73.23.-b, 74.45.+c, 74.50.+r

The rest of the paper is organized as follows. In Sec. II, we describe the model considered for a superconducting one-channel contact coupled to a generic electromagnetic environment; Sec. III is devoted to the analysis of the transition rates between different quasiparticles states induced by quantum phase fluctuations; in Sec. IV, we focus on the experimental situation of Ref. [15] and compare the theoretical results for the transition rates and the stationary probability for quasiparticles trapped in the subgap states with the corresponding experimental results. In Sec. V, we present our main conclusions. The more technical details on our calculations are described in Appendices A-D.

\section{THEORETICAL FRAMEWORK}

We consider the situation illustrated in Fig. 1(a) with a single superconducting channel (SC) coupled to an arbitrary impedance $Z_{\text {env }}$. The excitation spectrum of the SC contains a discrete, spin-degenerate Andreev level, with an energy $E_{A}(\delta)=\Delta \sqrt{1-\tau \sin ^{2} \delta / 2}$, where $\delta$ is the superconducting phase difference across the contact and $\tau$ the transmission probability for electrons [16] [see Fig. 1(b)]. The Andreev level is completely empty when the channel is in its ground state, which has a phase dependent energy $-E_{A}$ and carries a supercurrent $I=-\left(\partial E_{A} / \partial \delta\right) / \varphi_{0}$, where $\varphi_{0}=\hbar / 2 e$ is the reduced flux quantum. The lowest-energy excitations correspond to the occupation of the Andreev level by a single quasiparticle (of either spin), the global energy and the supercurrent of these "odd" configurations being then zero. There is also an excitation of energy $2 E_{A}$ with respect to the ground state, where the Andreev level is occupied by two quasiparticles of opposite spins. This "even" configuration can be seen as a localized excited "Andreev pair" [17], and carries a supercurrent opposite to that in the ground state.

The system Hamiltonian can be written as $\hat{H}=\hat{H}_{S C}(\hat{\delta})+$ $\hat{H}_{\text {env }}(\hat{\gamma})$, where the first term describes the $\mathrm{SC}$ and the second one the electromagnetic environment, modelled by the impedance $Z_{\text {env }}$. The phases $\delta$ and $\gamma$ across the SC 
(a)

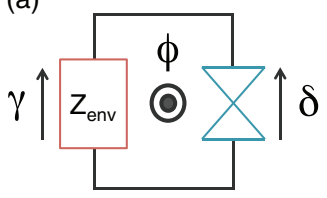

(b)

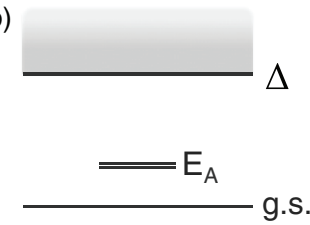

FIG. 1. (Color online) (a) Schematic representation of a single superconducting channel coupled to an impedance $Z_{\text {env }} . \delta$ and $\gamma$ indicate the phase drops through the channel and the impedance, respectively, and $\Phi$ is the magnetic flux through the loop. (b) Quasiparticle excitation spectrum in the single channel weak link, with the continuum above the gap $\Delta$ and a subgap discrete spin-degenerate Andreev level of energy $E_{A}$.

and the impedance are related by $\hat{\delta}-\hat{\gamma}=\Phi / \varphi_{0}=\varphi$, where $\Phi$ is the magnetic flux through the loop.

The population of the SC electronic states becomes then sensitive to the effects of quantum phase fluctuations. Assuming that $\operatorname{Re}\left(Z_{\text {env }}\right) \ll R_{Q}$, we treat the fluctuations to lowest order in perturbation and write the Hamiltonian $\hat{H}=\hat{H}_{\text {env }}(\hat{\gamma})+\hat{H}_{S C}(\varphi)+\varphi_{0} \hat{\gamma} \hat{I}(\varphi)$, where $\hat{I}=\varphi_{0}^{-1} \partial \hat{H}_{S C} / \partial \delta$ is the current operator in the contact region.

To describe the unperturbed single-channel SC we use a one-dimensional SNS junction model with a Dirac delta potential barrier (to account for nonperfect transmission) inside a normal region of negligible length. Details of the diagonalization of this model in terms of Bogoliubov fermion operators $\gamma_{\alpha, \sigma}$, where $\sigma$ indicates spin, are given in Appendix A. Two types of states are obtained, $\alpha \equiv k$ with energy $E_{k} \geqslant \Delta$ corresponding to the extended continuum states and $\alpha \equiv A$ corresponding to the localized Andreev states with energy $E_{A}$ [see Fig. 1(b)]. The SC ground state $\left|\Psi_{0}\right\rangle$ corresponds to the absence of excitations, i.e., $\gamma_{\alpha, \sigma}\left|\Psi_{0}\right\rangle=0$.

\section{TRANSITION RATES}

The coupling of the SC to the environment allows for transitions between different quasiparticle states. We shall first consider processes which permit the removal of a quasiparticle from the Andreev level. These processes allow in particular the relaxation of the lowest-energy excited states with one trapped quasiparticle back to the ground state $\left|\Psi_{0}\right\rangle$ [18]. They consist either in the absorption of an environmental photon and transfer of the trapped quasiparticle into the continuum states, or in the recombination of a quasiparticle from the continuum with the trapped one into a Cooper pair while releasing the energy as a photon. These two processes are illustrated in panels (a) and (b) of Fig. 2 and the corresponding rates are denoted by $\Gamma_{\text {out }}^{(a, b)}$. The Fermi golden rule for the first process yields

$$
\begin{aligned}
\Gamma_{\mathrm{out}}^{(a)}= & \frac{2 \pi}{\hbar} \sum_{k}\left|\left\langle k, \sigma\left|\varphi_{0} \hat{I}\right| A, \sigma\right\rangle\right|^{2} P\left[E_{k}-E_{A}(\delta)\right] \\
& \times\left[1-f_{\mathrm{FD}}\left(E_{k}, T_{\mathrm{qp}}\right)\right],
\end{aligned}
$$

where $f_{\mathrm{FD}}\left(E, T_{\mathrm{qp}}\right)$ is the Fermi population factor for quasiparticles in the continuum (assumed to be in equilibrium at a temperature $T_{\mathrm{qp}}$ ) and $P(E)$ is the probability of absorbing a photon of energy $E$ from the environment. This probability is $P(E)=D(E) f_{\mathrm{BE}}\left(E, T_{\text {env }}\right)$, where $f_{\mathrm{BE}}(E, T)$ is the Bose
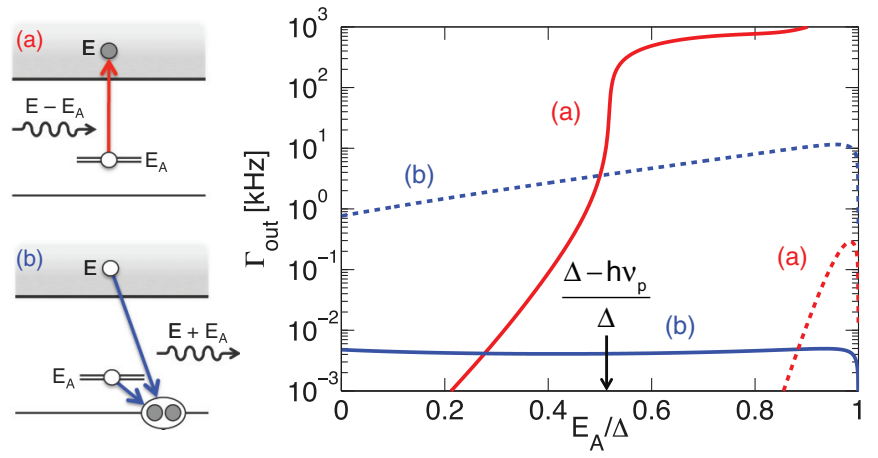

FIG. 2. (Color online) (Left) Schematic representation of processes removing a quasiparticle from the Andreev level. Process (a) involves absorption of an environmental photon or phonon and corresponds to the transition rate $\Gamma_{\text {out }}^{(a)}$. Process (b) corresponds to the recombination into a Cooper pair of the quasiparticle trapped in the Andreev level with a quasiparticle from the continuum, with the emission of a photon or a phonon, and is characterized by a transition rate $\Gamma_{\text {out }}^{(b)}$. The right panel shows the rates $\Gamma_{\text {out }}^{(a)}$ (red) and $\Gamma_{\text {out }}^{(b)}$ (blue) resulting from the absorption and emission of environmental photons (full lines) or phonons (dashed lines), for the parameters of Ref. [15] (see Appendix C). We have set $k_{B} T_{\text {env }}=0.06 \Delta, k_{B} T_{\mathrm{qp}}=0.09 \Delta$, and $k_{B} T_{p h}=0.015 \Delta$.

population factor, and $D(E)=\operatorname{Re}\left\{Z_{\text {env }}(E) / E\right\} / R_{Q}$, with $R_{Q}=h / 4 e^{2}$, is the density of states for the modes in the environment [20]. The environment is assumed to be in equilibrium at a temperature $T_{\text {env }}$, which can be, in general, different from $T_{\mathrm{qp}}$. The numerical evaluation of this rate (and every other) for different transmissions, shows a rather universal dependence in the Andreev level energy position $E_{A}$. Simple analytical expressions can be derived in the perfect transmission limit $\tau \rightarrow 1$ and in the tunnel limit $\tau \rightarrow 0$, for which the wave functions have a considerably simpler form. In this limit, one obtains (see Appendix B)

$$
\begin{aligned}
\Gamma_{\mathrm{out}}^{(a)}= & \frac{8 \Delta}{h} \int_{\Delta}^{\infty} d E D\left(E-E_{A}\right) g\left(E, E_{A}\right) \\
& \times f_{\mathrm{BE}}\left(E-E_{A}, T_{\mathrm{env}}\right)\left[1-f_{\mathrm{FD}}\left(E, T_{\mathrm{qp}}\right)\right],
\end{aligned}
$$

with $g\left(E, E_{A}\right)=\sqrt{\left(E^{2}-\Delta^{2}\right)\left(\Delta^{2}-E_{A}^{2}\right)} /\left[\Delta\left(E-E_{A}\right)\right]$. We also give in Appendix $\mathrm{B}$ the expression of $g\left(E, E_{A}\right)$ in the tunnel limit $\tau \rightarrow 0$.

When the environment of the SC contains a single mode with infinite quality factor, and at low temperature, this expression simplifies to $\Gamma_{\text {out }}^{(a)}=\frac{2 \Delta}{\hbar} \frac{Z_{0}}{R_{Q}} g\left(E_{A}+h v, E_{A}\right) \exp \left(-h v / k_{B} T_{\text {env }}\right), \quad$ where $v$ is the mode frequency. The function $g$ is of order 1 when $\Delta-h v<E_{A}<\Delta$, so that the rate is simply determined by the impedance $Z_{0}$ of the oscillator. For aluminum and for $Z_{0}=50 \Omega, \frac{2 \Delta}{\hbar} \frac{Z_{0}}{R_{Q}} \approx 1 \mathrm{GHz}$.

In a similar way for the second relevant process, we find

$$
\begin{aligned}
\Gamma_{\mathrm{out}}^{(b)}= & \frac{8 \Delta}{h} \int_{\Delta}^{\infty} d E D\left(E+E_{A}\right) g\left(E,-E_{A}\right) \\
& \times\left[1+f_{\mathrm{BE}}\left(E+E_{A}, T_{\mathrm{env}}\right)\right] f_{\mathrm{FD}}\left(E, T_{\mathrm{qp}}\right) .
\end{aligned}
$$

We show in Appendix D that for perfect transmission the matrix elements for electron-phonon coupling have the same 

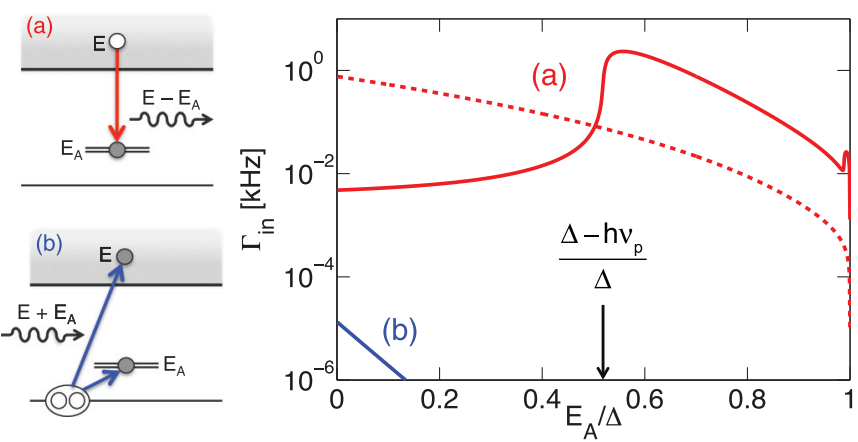

FIG. 3. (Color online) (Left) Schematic representation of processes adding a quasiparticle in the Andreev level. (Right) Emission processes (a) rates, denoted by $\Gamma_{\mathrm{in}}^{(a)}$ in the text, are given by the red curves for photons (full line) and phonons (dashed lines). The processes (b) involving the breaking of a Cooper pair (blue line in the plot) are much less efficient. The one involving the phonons is below this scale. Same parameters as in Fig. 2.

functional form in terms of $E$ and $E_{A}$ as those for the coupling with the electromagnetic modes. Therefore its inclusion leads to the same expressions for $\Gamma_{\text {out }}^{(a, b)}$ as in Eqs. (2) and (3) but with a quadratic density of states and $T_{\text {env }}$ replaced by the phonon temperature $T_{p h}$ (see Appendix D for more details). The time reversed processes, illustrated in Fig. 3 and characterized by rates $\Gamma_{\text {in }}^{(a)}$ and $\Gamma_{\text {in }}^{(b)}$ are responsible for the population of the Andreev level, either by trapping a quasiparticle from the continuum or by breaking a pair.

\section{COMPARISON TO EXPERIMENTS}

We focus on the recent experiments on superconducting atomic contacts [15] that have analyzed in detail the quasiparticle trapping in Andreev levels and its dynamics. In these experiments, an atomic contact was embedded in a superconducting loop containing a Josephson junction, thus forming an asymmetric superconducting quantum interference device (SQUID). It was found that there is a significant probability for the SC to get trapped in an odd state in which the highest transmitted channel carries no supercurrent. The experiments also showed that the relaxation rates for these states fall into a nearly universal behavior as a function of the energy $E_{A}$ regardless of the particular values of the transmission and phase difference. Trapping occurred essentially when the Andreev level energy was smaller than half the superconducting gap $E_{A} \lesssim 0.5 \Delta$, with the lifetime of trapped quasiparticles exceeding $100 \mu$ s. For larger energies, no significant trapping could be detected. The origin of this sharp energy threshold was a puzzle not explained in the paper reporting the experiment.

In Ref. [15], the SQUID Josephson junction had a Josephson energy much larger than the charging energy, and it can therefore be described as an harmonic oscillator. Spectroscopy measurements [17,21] on similar circuits as the one used in Ref. [15] showed that the plasma frequency of this mode can be significantly renormalized by parallel inductances and approach $\sim 0.5 \Delta$ (see Appendix $C$ and Ref. [21]). As explained in the following, our theory shows that the main relaxation mechanism for the trapped quasiparticle states is their excitation into the extended continuum states above the superconducting gap by absorption of photons from the plasma mode. This mechanism becomes inefficient when the energy difference between the Andreev level and the continuum exceeds the plasma energy, $\Delta-E_{A}>h v_{p}$, hence providing a simple explanation for the observed behavior.

The results for the transition rates $\Gamma_{\text {out }}^{(a, b)}$ obtained using parameters which are appropriate for the experimental situation of Ref. [15] (see Appendix C) are shown by the solid lines in Fig. 2. For $E_{A} \gtrsim \Delta-h v_{p} \sim 0.52 \Delta, \Gamma_{\text {out }}^{(a)}$ is large because photons in the plasma mode can excite the trapped quasiparticle out into the continuum. Similarly, $\Gamma_{\text {in }}^{(a)}$ is large in this energy range because quasiparticles near the gap edge can relax in the Andreev level while emitting a plasma photon (see Fig. 3). For lower energies, the energy of the plasma photons is not sufficient and the rate drops abruptly. Other processes, like phonon absorption or emission start to play a role. Hence, both $\Gamma_{\text {in }}$ and $\Gamma_{\text {out }}$ are determined by phonon processes for $E_{A}<\Delta-h v_{p}$ and by photon processes for $E_{A}>\Delta-h v_{p}$. It should be noticed that three different temperatures enter the calculation. We assume that the phonons in the Al films of Ref. [15] are at equilibrium with the substrate and therefore $T_{p h}$ is taken equal to the base temperature measured by the thermometers in the experiment $(30 \mathrm{mK})$. The two other temperatures, $T_{\mathrm{env}}$ and $T_{\mathrm{qp}}$, can be significantly larger due to incomplete filtering of radiation. To fit the results we have used $T_{\text {env }} \sim 120 \mathrm{mK}$, similar to what is deduced from measurements of the switching probability of the SQUID [21,22] and $T_{\mathrm{qp}} \sim 180 \mathrm{mK}$ which simulates the presence of a few tens of out-of-equilibrium quasiparticles per $\mu \mathrm{m}^{3}$, as typically found in experiments with $\mathrm{Al}$ resonators and qubits [2-4,6,23].

The different transitions which determine the population and relaxation of the Andreev level are illustrated in the inset of Fig. 4. They also involve the even excited state $\mid$ even* $\left.{ }^{*}\right\rangle=\gamma_{A \uparrow}^{\dagger} \gamma_{A \downarrow}^{\dagger}\left|\Psi_{0}\right\rangle$. The analysis is further simplified by symmetry relations: the rates connecting the even excited state and the odd states are equal to the ones connecting the odd states and the even ground state. This is indicated by the color code used for the arrows in the inset of Fig. 4. Notice that the full determination of the level populations requires also the evaluation of the rates $\Gamma_{e^{*} \rightarrow e}$ and $\Gamma_{e \rightarrow e^{*}}$. In Ref. [15], it was assumed that the relaxation rate $\Gamma_{e^{*} \rightarrow e}$ to the ground state was very fast compared to all other ones and that $\Gamma_{e \rightarrow e^{*}}$ was negligible. For photonic or phononic environments, these have been calculated in Refs. [10,20] and [24], respectively, and reproduced with the present formalism, as discussed in Appendix B and D. The calculation does corroborate that $\Gamma_{e^{*} \rightarrow e}$ is much larger than all the other rates for the transmissions explored in the experiment.

A last step in our calculation is to obtain the stationary distribution of quasiparticles by solving the master equation involving all transitions indicated in the inset of Fig. 4. The result for the occupation probability of the odd states, $P_{\infty}$, is shown in Fig. 4 and compared with the experimental results from Ref. [15] for contacts with different transmissions. As can be observed, the theory qualitatively describes the decrease in $P_{\infty}$ at fixed $E_{A}$, which is observed experimentally for increasing transmission in the slow-relaxation regime. There 


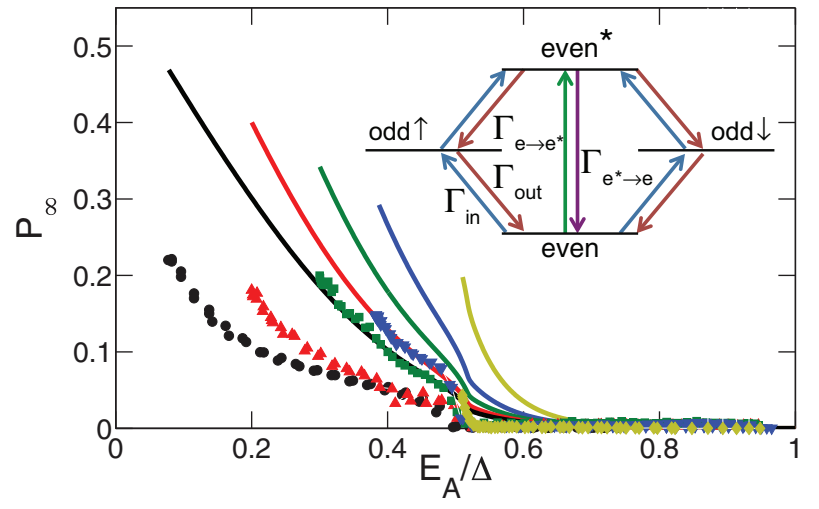

FIG. 4. (Color online) Stationary occupation probability $P_{\infty}$ for the odd states, using parameters of Ref. [15]. The theoretical results (full lines) are compared with the experimental results for different values of the contact transmission $\tau=0.994$ (full circles, black); 0.96 (upper triangles, red); 0.91 (squares, dark green); 0.85 (down triangles, blue) and 0.74 (diamonds, light green). Same parameters as in Figs. 2 and 3. (Inset) Scheme of the Andreev level occupation configurations and the different transitions induced by the coupling to the environment. The total rates $\Gamma_{\text {out,in }}$ connecting odd states with the even states (red and blue arrows) are obtained by adding $\Gamma_{\text {out,in }}^{(a)}$ and $\Gamma_{\text {out,in. }}^{(b)}$. The rates $\Gamma_{e^{*} \rightarrow e}$ and $\Gamma_{e^{*} \rightarrow e}$ connecting the even states (green and purple arrows) are calculated within the same model (see Appendix B).

is, however, some discrepancy in the quantitative values of $P_{\infty}$ which is overestimated in our model calculations.

We show in Fig. 5 the comparison of the experimental and theoretical results for the total rates $\Gamma_{\text {in }}$ and $\Gamma_{\text {out }}$ as a function of $E_{A}$ for different values of the contact transmission. One should remark the quite good agreement which is obtained for $\Gamma_{\text {out }}$ in the fast-relaxation regime $\left(E_{A}>0.5 \Delta\right)$ and for $\Gamma_{\text {in }}$ in the slow-relaxation regime. The drop in $\Gamma_{\text {out }}$ by more than two orders of magnitude at $E_{A} \sim 0.5 \Delta$ is also captured by our model. In the slow-relaxation regime the model correctly describes the decrease of $\Gamma_{\text {in }}$ and the increase in $\Gamma_{\text {out }}$, which is observed at fixed $E_{A}$ with increasing transmission. The largest

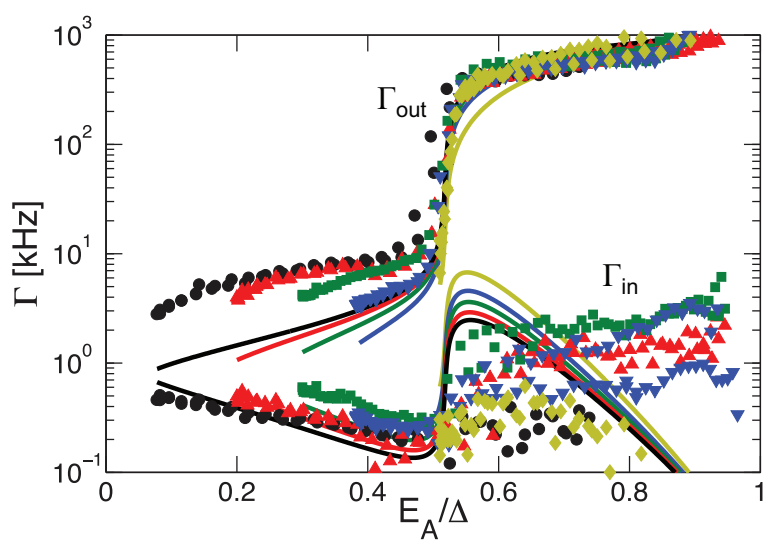

FIG. 5. (Color online) Total rates $\Gamma_{\text {out }}$ (upper curves and symbols) and $\Gamma_{\text {in }}$ (lower cuves and symbols) calculated by the present model and obtained experimentally in Ref. [15] for different contact transmissions. The same convention as in Fig. 4 is used. discrepancies between model and experiment are found for $\Gamma_{\text {in }}$ when $E_{A} \gtrsim 0.5 \Delta$ and for $\Gamma_{\text {out }}$ when $E_{A} \lesssim 0.5 \Delta$. It should be noticed, however, that the experimental determination of $\Gamma_{\text {in }}$ is less precise for $E_{A}>0.5 \Delta$, where $P_{\infty} \ll 1$. The decay of $\Gamma_{\text {in }}$ in theory is essentially related to the assumption of a thermal distribution of quasiparticles, which leads to $\Gamma_{\text {in }} \propto$ $\exp \left[-\left(E_{A}+h v_{p}\right) / k_{B} T_{q p}\right]$. A weaker dependence on energy would be obtained with a self-consistent description of this distribution [3]. On the other hand, the deviations found for $\Gamma_{\text {out }}$ in the slow-relaxation regime are more significant and could indicate that some additional relaxation mechanism, like tunneling to vortices or quasiparticle traps in the vicinity of the contact, could be contributing for small $E_{A}$. Since in the limit where $\Gamma_{e^{*} \rightarrow e} \gg \Gamma_{\text {in }}, \Gamma_{\text {out }} \gg \Gamma_{e \rightarrow e^{*}}$, the stationary occupation probability $P_{\infty}$ is simply given by $2 \Gamma_{\text {in }} /\left(3 \Gamma_{\text {in }}+\Gamma_{\text {out }}\right)$ [22], the deviations for $\Gamma_{\text {out }}$ explain why the theory overestimates $P_{\infty}$ in this regime. Further combined experimental and theoretical work would be required to clarify this point.

\section{CONCLUSIONS}

In conclusion, we have presented a theory which describes the dynamics of trapping and untrapping quasiparticles in phase-biased superconducting weak links. It is shown that in realistic conditions this dynamics can be controlled by the coupling of the weak link to its electromagnetic environment. The results are in semiquantitative agreement with the experiments of Ref. [15], where the sharp jump observed in the trapping and untrapping rates is associated to the onset of the coupling to the environment plasma mode. The mechanisms described here can be relevant for controlling decoherence in superconducting qubits involving channels with non-negligible transmission. In case of the Andreev qubits discussed in Refs. [10,11], where poisoning by trapped quasiparticles in the ABS should be avoided, the presence of a mode of energy larger than $\Delta-E_{A}$ would be beneficial. In contrast, for the proposals of Refs. [12,13], which are based on the manipulation of the odd states, a larger lifetime of the trapped quasiparticles is desirable. In this case, one would need an electromagnetic environment containing no mode of frequency larger than $\Delta-E_{A}$. Finally, as discussed in Ref. [5], even in the case of qubits based on tunnel junctions changes in the occupation of the Andreev states make the Josephson coupling and hence the qubit frequency fluctuate thus giving rise to dephasing. Therefore, even in this case of Andreev levels very close to the gap edge, slowing down the dynamics of these occupations could have an influence on the qubit decoherence.

\section{ACKNOWLEDGMENTS}

The authors acknowledge fruitful discussions with J. Martinis, R. Egger, A. Zazunov, D. Urban, J. C. Cuevas, F. S. Bergeret, and A. Martín-Rodero. Financial support by EU FP7 SE2ND project, Spanish Mineco project FIS2011-26516, ANR contracts DOCFLUC and MASH, C'Nano and by the People Programme (Marie Curie Actions) of the European Union's Seventh Framework Programme (FP7/2007-2013) under REA grant agreement No. PIIF-GA-2011-298415 is acknowledged. 


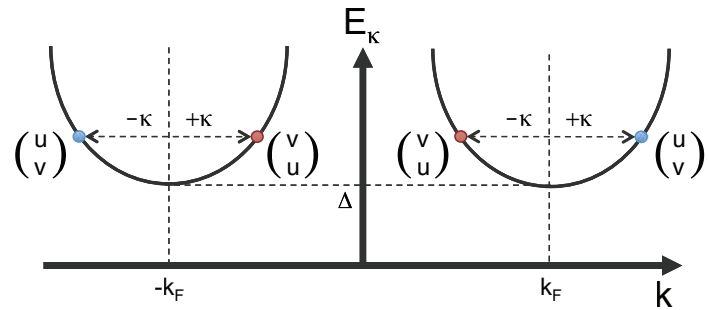

FIG. 6. (Color online) Quasiparticle energies and twocomponent eigenfunctions near the Fermi level for spin-up quasiparticles. There is a four-fold degeneracy of states for each energy $E$, with two quasielectron states (blue dots) with a momentum $|k|=k_{F}+\kappa_{E}$ and another two quasiholes with an absolute momentum of $|k|=k_{F}-\kappa_{E}$.

\section{APPENDIX A: DIAGONALIZATION OF THE SC HAMILTONIAN AND SC WAVE FUNCTIONS}

The point contact is modeled as a 1D SNS junction with a Dirac delta barrier in the normal region. The normal region's length $\tilde{L}_{N}$ can be taken to the limit $\tilde{L}_{N} \rightarrow 0$ in the ballistic regime, effectively turning the scattering problem into the problem of a delta barrier in a superconducting system, with a well-defined phase bias between the left and right leads.

Continuum wave functions. For an homogeneous superconducting system, the Hamiltonian's eigenfunctions have a momentum $|k|=k_{F} \pm \kappa_{E}$, where $\kappa_{E}=\xi_{0}^{-1} \sqrt{(E / \Delta)^{2}-1}\left(\xi_{0}\right.$ being the superconducting coherence length, given by $h v_{F} / \Delta$ in the ballistic regime). The eigenfunctions with positive energy $E_{k}$ and spin-up take the following shape:

$$
\begin{aligned}
& \psi_{k, \tilde{\varphi}}^{(\uparrow)}(x)=\frac{1}{\sqrt{L}}\left(\begin{array}{cl}
u_{E} \\
v_{E} e^{i \tilde{\varphi}}
\end{array}\right) e^{+i k x} \quad \begin{array}{l}
\text { for }|k|>k_{F} \\
\text { (quasielectrons) }
\end{array}, \\
& \psi_{k, \tilde{\varphi}}^{(\uparrow)}(x)=\frac{1}{\sqrt{L}}\left(\begin{array}{cc}
v_{E} \\
u_{E} e^{i \tilde{\varphi}}
\end{array}\right) e^{-i k x} \quad \begin{array}{l}
\text { for }|k|<k_{F} \\
\text { (quasiholes) }
\end{array},
\end{aligned}
$$

where $\tilde{\varphi}$ is the phase of the superconducting order parameter, $L$ is a length over which the freely propagating eigenfunctions are defined and $u_{E}, v_{E}$ are, respectively, the electron and hole components of the plane wave:

$$
(u / v)_{E}=\frac{1}{\sqrt{2}} \sqrt{1 \pm \sqrt{1-(\Delta / E)^{2}}} .
$$

These plane waves are schematically shown in Fig. 6 .

Summations in momenta such as the one in Eq. (1) may be rewritten, for the sake of convenience, as integrals over quasiparticle energies weighted by the superconducting density of states $\rho_{\mathrm{SC}}(E)=\rho_{F} \frac{|E|}{\sqrt{E^{2}-\Delta^{2}}}$, where $\rho_{F}$ is the normal density of states at the Fermi level. That being the case we shall brand the wave functions (and the states they refer to) not by using their momentum $k$ as an index, but their energy $E_{k} \rightarrow E$, their associated quasielectron/hole character and the direction of their momentum. With the plane waves from Eq. (A1) it is possible to construct solutions to the BdeG equations in an inhomogeneous system following a scattering approach [25].

These wave functions are Nambu spinors of the form

$$
\psi_{E \uparrow}^{(\eta \pm)}(x)=\left(\begin{array}{l}
U_{E}^{(\eta \pm)}(x) \\
V_{E}^{(\eta \pm)}(x)
\end{array}\right)
$$

where $U_{E}$ is the electron amplitude, $V_{E}$ is the hole amplitude, and $\eta$ denotes the electron/hole character of the quasiparticle state. Spin-down eigenfunctions can be easily obtained from spin-up ones by use of the electron-hole symmetry in the system, through the substitution $U \rightarrow V^{*}, V \rightarrow-U^{*}$. For the sake of simplicity, we shall omit the spin sub-index in the wave functions that we discuss next.

Condensing all the phase difference $\delta$ in the right lead, the wave functions take the following shape:

$$
\begin{aligned}
\psi_{E}^{(\eta \pm)}(x)= & \psi_{\mathrm{Src}}^{(\eta \pm)}(x) \\
& +\left[A_{(\eta \pm)} \psi_{E, 0}^{(e-)}(x)+B_{(\eta \pm)} \psi_{E, 0}^{(h-)}(x)\right] \Theta(-x) \\
& +\left[C_{(\eta \pm)} \psi_{E, \delta}^{(e+)}(x)+D_{(\eta \pm)} \psi_{E, \delta}^{(h+)}(x)\right] \Theta(x),
\end{aligned}
$$

where $\psi_{\mathrm{Src}}^{(\eta \pm)}$ is a source term of a quasielectron or quasihole impinging the contact from any of the leads:

$$
\begin{aligned}
& \psi_{\mathrm{Src}}^{(e+)}=\psi_{E, 0}^{(e+)}(x) \Theta(-x), \quad \psi_{\mathrm{Src}}^{(h+)}=\psi_{E, 0}^{(h+)}(x) \Theta(-x), \\
& \psi_{\mathrm{Src}}^{(e-)}=\psi_{E, \delta}^{(e-)}(x) \Theta(x), \quad \psi_{\mathrm{Src}}^{(h-)}=\psi_{E, \delta}^{(h-)}(x) \Theta(x) .
\end{aligned}
$$

The rest of the contributions to the wave functions are outgoing partial waves (as illustrated in Fig. 7).

Imposing continuity for the wave function and its derivative (taking into account the effect of the $\delta$ barrier) the values of the partial wave coefficients are obtained for each incidence:

$$
\begin{aligned}
& A_{(e+)}=-\mu_{\tau} \tau\left(\mu_{\tau}+i\right) \sinh ^{2} \theta_{E} Q^{-1}, \\
& B_{(e+)}=i \tau \sin \delta / 2 \sinh \left(\theta_{E}+i \frac{\delta}{2}\right) Q^{-1}, \\
& C_{(e+)}=-i \tau\left(\mu_{\tau}+i\right) e^{-i \delta / 2} \sinh \theta_{E} \sinh \left(\theta_{E}+i \frac{\delta}{2}\right) Q^{-1}, \\
& D_{(e+)}=-\mu_{\tau} \tau e^{-i \delta / 2} \sinh \theta_{E} \sin \frac{\delta}{2} Q^{-1}, \\
& A_{(e-)}=C_{(e+)}(-\delta), \quad A_{(h+)}=B_{(e+)}^{*}, \quad A_{(h-)}=C_{(h+)}(-\delta), \\
& B_{(e-)}=D_{(e+)}(-\delta), \quad B_{(h+)}=A_{(e+)}^{*}, \quad B_{(h-)}=D_{(h+)}(-\delta), \\
& C_{(e-)}=A_{(e+)}(-\delta), \quad C_{(h+)}=D_{(e+)}^{*}, \quad C_{(h-)}=A_{(h+)}(-\delta), \\
& D_{(e-)}=B_{(e+)}(-\delta), \quad D_{(h+)}=C_{(e+)}^{*}, \quad D_{(h-)}=B_{(h+)}(-\delta),
\end{aligned}
$$

(a)

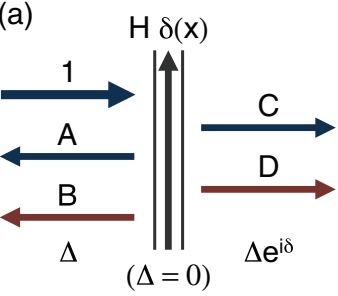

(b)

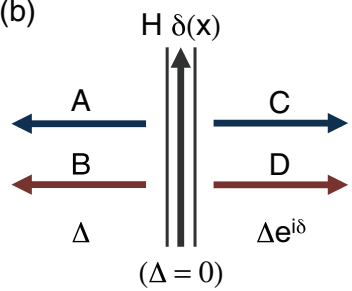

FIG. 7. (Color online) Schematic representation of the scattering problem for the case of a quasi-electron impinging the contact and the resulting outgoing quasiparticle partial waves (a), either normally or Andreev-reflected or transmitted. The scattering problem for the ABS does not require a source term (b), but these outgoing partial waves exhibit an evanescent behavior. 


$$
\begin{aligned}
& \left.\Delta \stackrel{E, \eta \pm, \sigma}{\bigcirc} \gamma_{E, \eta \pm \sigma}^{+}\left|\Phi_{0}\right\rangle=\mid \text { Even; } E, \eta \pm, \sigma\right\rangle \\
& \begin{array}{r}
\left.\mathrm{E}_{\mathrm{A}}(\phi)=\gamma_{\mathrm{A} \gamma}^{+} \gamma_{A \downarrow}^{+}\left|\Phi_{0}\right\rangle=\mid \text { Even }^{*}\right\rangle \\
\left.\gamma_{\mathrm{A \sigma}}^{+}\left|\Phi_{0}\right\rangle=\mid \text { Odd, } \sigma\right\rangle \\
\left.\left|\Phi_{0}\right\rangle=\mid \text { Even }\right\rangle
\end{array}
\end{aligned}
$$

FIG. 8. Single-particle excitations of the unperturbed SC Hamiltonian, with the parity notation for different ABS occupancies. The ground state of the system is noted as the even state. As single-particle excitations of the ABS have an odd parity, the ABS doublet excitation has an even parity, so it is branded as the even excited $\mid$ Even $\left.^{*}\right\rangle$ state.

where $\sinh \theta_{E}=\sqrt{\left(\frac{E}{\Delta}\right)^{2}-1}, \quad \mu_{\tau}=\sqrt{\frac{R}{\tau}}, \tau$ is the normal transmission probability from the potential barrier, $R=1-\tau$ its normal reflection probability, and $Q=\sinh ^{2} \theta_{E}+\tau \sin ^{2} \frac{\delta}{2}$.

Andreev bound-state wave function. The wave amplitudes for states with $E<\Delta$ exhibit subgap poles at $E=E_{A}(\delta)=$ $\Delta \sqrt{1-\tau \sin ^{2} \frac{\delta}{2}}$, which signals the existence of a bound state at such an energy. The wave functions for the Andreev bound states (ABS) may be obtained in a similar way than those for states lying at $E>\Delta$ taking into account that the quasiparticle momentum gains an imaginary component below the gap. The partial waves for the ABS are

$$
\begin{aligned}
& \psi_{E_{A}, \tilde{\varphi}}^{(e \pm)}(x)=\frac{1}{\sqrt{\xi_{0}}}\left(\begin{array}{c}
u_{A} \\
v_{A} e^{i \tilde{\varphi}}
\end{array}\right) e^{ \pm i\left(k_{F}+i \kappa_{A}\right) x}, \\
& \psi_{E_{A}, \tilde{\varphi}}^{(h \pm)}(x)=\frac{1}{\sqrt{\xi_{0}}}\left(\begin{array}{c}
v_{A} \\
u_{A} e^{i \tilde{\varphi}}
\end{array}\right) e^{\mp i\left(k_{F}-i \kappa_{A}\right) x} .
\end{aligned}
$$

These differ from the propagating partial waves because the $u_{E}, v_{E}$ coefficients and the quasiparticle momentum $\kappa_{E}$ become complex for $E<\Delta$ :

$$
\begin{aligned}
u_{E} \rightarrow u_{A} & =\frac{1}{\sqrt{2}} e^{i \theta_{A} / 2}, \quad v_{E} \rightarrow v_{A}=\frac{1}{\sqrt{2}} e^{-i \theta_{A} / 2}, \\
\kappa_{E} \rightarrow i \kappa_{A} & =i \xi_{0}^{-1} \sin \theta_{A}, \\
\text { with } \sin \theta_{A} & =\sqrt{\tau}\left|\sin \frac{\delta}{2}\right|=\frac{\sqrt{\Delta^{2}-E_{A}^{2}}}{\Delta} .
\end{aligned}
$$

Only partial waves confined within a length $\kappa_{A}^{-1}$, which diverges for $E_{A} \rightarrow \Delta$, may appear in the wave functions

$$
\begin{aligned}
\psi_{A}(x)= & {\left[A_{A} \psi_{E_{A}, 0}^{(e-)}(x)+B_{A} \psi_{E_{A}, 0}^{(h-)}(x)\right] \Theta(-x) } \\
& +\left[C_{A} \psi_{E_{A}, \delta}^{(e+)}(x)+D_{A} \psi_{E_{A}, \delta}^{(h+)}(x)\right] \Theta(x) .
\end{aligned}
$$

A linear homogeneous system of equations is obtained for the partial wave weights by applying the same conditions as in the case of the continuum states. The system exhibits a nontrivial solution for $E=E_{A}(\delta)$. Eliminating the redundant equation and imposing the normalisation condition for the

wave function, it is finally obtained that, in the bound states

$$
\left\{\begin{array}{l}
A_{A}=-i \tilde{N}_{A} \sin \left(\theta_{A}-\frac{\delta}{2}\right) e^{-i \tilde{\beta}_{\tau}} e^{i \delta / 2} \\
B_{A}=-i \sqrt{R} \tilde{N}_{A} \sin \frac{\delta}{2} \cdot e^{i \tilde{\beta}_{\tau}} e^{i \delta / 2} \\
C_{A}=\sqrt{R} \tilde{N}_{A}\left|\sin \frac{\delta}{2}\right| e^{-i \tilde{\beta}_{\tau}} \\
D_{A}=\sigma_{\delta} \tilde{N}_{A} \sin \left(\theta_{A}-\frac{\delta}{2}\right) e^{i \tilde{\beta}_{\tau}}
\end{array}\right.
$$

where $\sin \tilde{\beta}_{\tau}=\sqrt{\tau}, \sigma_{\delta}=\operatorname{sign}(\delta)$ and

$$
\tilde{N}_{A}=\sqrt{\frac{-\sigma_{\delta} \sqrt{\tau}}{2 \cos \theta_{A} \sin \left(\theta_{A}-\frac{\delta}{2}\right)}} .
$$

A similar derivation of this result can be found in Ref. [20].

The solutions of the BdeG equations allow us to express the electron field operators as

$$
\begin{aligned}
\Psi_{\sigma}(x)= & U_{A}(x) \gamma_{A \sigma}-\sigma V_{A}^{*}(x) \gamma_{A \bar{\sigma}}^{\dagger} \\
& +\sum_{E, \eta \pm}\left[U_{E}^{(\eta \pm)}(x) \gamma_{E, \eta \pm, \sigma}-\sigma V_{E}^{(\eta \pm) *}(x) \gamma_{E, \eta \mp, \bar{\sigma}}^{\dagger}\right]
\end{aligned}
$$

where $\gamma_{A \sigma}$ and $\gamma_{E, \eta \pm, \sigma}$ are the quasiparticle operators that diagonalize the SC Hamiltonian. The excitation spectrum of the system is represented in Fig. 8.

\section{APPENDIX B: CURRENT OPERATOR AND TRANSITION RATES} is

The current operator in the new basis defined by Eq. (A15)

$$
\hat{I}(x)=-\frac{\hbar e}{2 m i} \sum_{i, j, \sigma}\left(\gamma_{i \sigma}^{\dagger} \gamma_{i \bar{\sigma}}\right)\left(\begin{array}{cc}
U_{i}^{*} \frac{d U_{j}}{d x}-\frac{d U_{i}^{*}}{d x} U_{j} & \sigma\left(U_{i}^{*} \frac{d V_{-j}^{*}}{d x}-\frac{d U_{i}^{*}}{d x} V_{-j}^{*}\right) \\
\sigma\left(V_{-i} \frac{d U_{j}}{d x}-\frac{d V_{-i}}{d x} U_{j}\right) & V_{-i} \frac{d V_{-j}^{*}}{d x}-\frac{d V_{-i}}{d x} V_{-j}^{*}
\end{array}\right)\left(\begin{array}{c}
\gamma_{j \sigma} \\
\gamma_{j \bar{\sigma}}^{\dagger}
\end{array}\right) .
$$

The sum in $i, j$ indices are a shorthand notation for all the different contributions appearing in Eq. (A15). The minus sign in front of some particular wave-function subindices notes that such a component corresponds to the antiparallel wave function [e.g., if $i=E(e+)$, then $U_{-i}=U_{E}^{(e-)}$ ], which only applies when the index corresponds to an excitation in the continuum.

\section{Transitions involving the odd states}

A relevant matrix element in the problem is the one associated to the $\mid$ Odd,$\sigma\rangle \rightarrow \mid$ Even; $E, e+, \sigma\rangle$ process, which is found to be

$$
-\frac{\hbar e k_{F}}{m \sqrt{L \xi_{0}}}\left(C_{A} C_{(e+)}^{*}-D_{A} D_{(e+)}^{*}\right)\left(u_{E} u_{A}+v_{E} v_{A}\right)
$$

The electron-hole symmetry in the field transformations ensures that this matrix element is the complex conjugate of the matrix element associated to the process $\mid$ Odd, $\sigma\rangle \rightarrow$ $\mid$ Even; $E, h-, \sigma\rangle$. 
In the ballistic limit $\tau \rightarrow 1$, restricting $\delta$ to the interval $[0, \pi]$ so as to establish a bijection between $\delta$ and $E_{A}(\delta)$, one finds

$$
\begin{gathered}
\left|C_{A}\right| \rightarrow\left(\frac{\Delta^{2}-E_{A}^{2}}{\Delta^{2}}\right)^{1 / 4}, \quad D_{A} \rightarrow 0, \\
\left|C_{(e+)}\right| \rightarrow \sqrt{\frac{E^{2}-\Delta^{2}}{E^{2}-E_{A}^{2}}}, \quad D_{(e+)} \rightarrow 0 .
\end{gathered}
$$

The squared amplitude of these matrix elements in this limit is

$$
\frac{\hbar^{2} e^{2} k_{F}^{2}}{m^{2} L \xi_{0}} \frac{\sqrt{\Delta^{2}-E_{A}^{2}}}{\Delta} \frac{E^{2}-\Delta^{2}}{E^{2}-E_{A}^{2}}\left(1+\frac{E_{A}}{E}\right) .
$$

Matrix elements for the $\mid$ Odd,$\sigma\rangle \rightarrow \mid$ Even; $E, e-, \sigma\rangle$ and $\mid$ Odd,$\sigma\rangle \rightarrow \mid$ Even; $E, h+, \sigma\rangle$ processes vanish in the limit of perfect transmission.

Conversely, the squared amplitude for the quasiparticle recombination processes $\mid$ Odd, $\sigma ; E, e-, \bar{\sigma}\rangle \rightarrow \mid$ Even $\rangle$ and $\mid$ Odd, $\sigma ; E, h+, \bar{\sigma}\rangle \rightarrow \mid$ Even $\rangle$ in the limit $\tau \rightarrow 1$ is found to be

$$
\frac{\hbar^{2} e^{2} k_{F}^{2}}{m^{2} L \xi_{0}} \frac{\sqrt{\Delta^{2}-E_{A}^{2}}}{\Delta} \frac{E^{2}-\Delta^{2}}{E^{2}-E_{A}^{2}}\left(1-\frac{E_{A}}{E}\right) .
$$

Whereas the amplitudes for the other two recombination processes, which are $\mid$ Odd,$\sigma ; E, e+, \bar{\sigma}\rangle \rightarrow \mid$ Even $\rangle$ and $\mid$ Odd, $\sigma ; E, h-, \bar{\sigma}\rangle \rightarrow \mid$ Even $\rangle$, are zero in this same limit.

The products of the terms that contain in these expressions the functional dependence in $E$ and $E_{A}$ with the superconducting density of states yield the factors $g\left(E, E_{A}\right)$ mentioned in the main text:

$$
g\left(E, E_{A}\right)=\frac{\sqrt{\left(E^{2}-\Delta^{2}\right)\left(\Delta^{2}-E_{A}^{2}\right)}}{\Delta\left(E-E_{A}\right)} .
$$

In the opposite tunnel limit $\tau \rightarrow 0$, all squared amplitudes tend to zero as $\tau^{3 / 2}$, with the leading term being the same for the four different processes:

$$
\frac{\hbar^{2} e^{2} k_{F}^{2}}{m^{2} L \xi_{0}} \frac{\tau}{2} \frac{\sqrt{\Delta^{2}-E_{A}^{2}}}{\Delta}\left(1+\frac{\Delta \cos \delta}{E}\right) .
$$

The same applies to amplitudes of recombination processes, with a minus sign appearing inside the parenthesis instead of a plus.

From these, we may define another $g\left(E, E_{A}\right)$ factor for the tunnel regime:

$$
g\left(E, E_{A}\right)=\frac{\tau}{2} \sqrt{\frac{\Delta^{2}-E_{A}^{2}}{E^{2}-\Delta^{2}}}\left[\frac{E}{\Delta}+\operatorname{sgn}\left(E_{A}\right) \cos \delta\right] .
$$

These results are in agreement with the ones recently derived by Kos et al. using a different method [19] taking also

into account the factor $\left(E \pm E_{A}\right)^{-1}$ that comes from the environmental density of states [see Eq. (C1)].

\section{Transitions between the even states}

An analytical expression for the amplitude that links the two even states can be derived for any value of $\tau$ :

$$
\begin{aligned}
\left\langle\text { Even }|\hat{I}| \text { Even }^{*}\right\rangle & =\frac{\hbar e k_{F}}{m \xi_{0}} C_{A} D_{A}\left(u_{A}^{2}-v_{A}^{2}\right) e^{i \delta}, \\
\mid\left.\left\langle\text { Even }|\hat{I}| \text { Even }^{*}\right\rangle\right|^{2} & =\frac{e^{2} \Delta^{4}(1-\tau) \tau^{2} \sin ^{4} \frac{\delta}{2}}{\hbar^{2}} .
\end{aligned}
$$

These results coincide with the results from [11].

\section{APPENDIX C: MODELLING THE EM ENVIRONMENT FOR THE EXPERIMENT IN REF. [1]}

The density of environmental modes $D(h v)$ is, following the formalism presented in Ref. [20],

$$
D(h v)=\frac{1}{h v} \frac{\operatorname{Re}\left\{Z_{\text {env }}(v)\right\}}{R_{Q}},
$$

where $Z_{\text {env }}(v)$ is the electric impedance as seen from the SC, represented in Fig. 9(c).

In the experiment shown in Ref. [15], a SC is placed in parallel with a Josephson junction having a critical current much larger than that of the atomic contact [see Fig. 9(b)]. This junction is perceived by the atomic contact as the parallel combination of an inductor $L_{J}=\varphi_{0} / I_{0}$ and a capacitor $C_{J}$, $I_{0}$ being the critical current of the junction. The finite quality factor of the corresponding electromagnetic ("plasma") mode is modeled with a resistance $R_{J}$ in series with the capacitor. The SQUID loop formed by the contact and the junction is

(a) $\quad \mathrm{L}_{\mathrm{e}}$

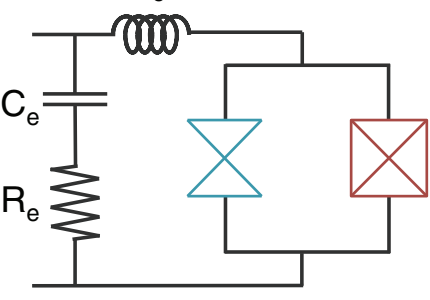

(c)

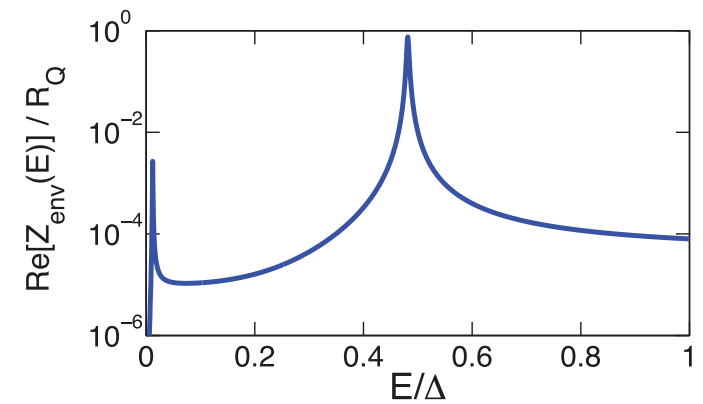

FIG. 9. (Color online) (a) Electrical elements in the relevant neighborhood of the SQUID loop. (b) Equivalent electrical model for the Josephson junction in the SQUID. (c) Environmental impedance as seen by the SC. 
connected to a biasing circuit through an inductor $L_{e}$ and a capacitance $C_{e}$. Dissipation in this circuit is modelled by a resistance $R_{e}$ in series with the capacitor [see Fig. 9(a)].

The total impedance seen by the SC is

$$
\frac{Z_{\mathrm{env}}(v)}{R_{Q}}=\frac{4}{\pi} \frac{E_{C}}{h v_{p 0}} \frac{i \frac{v}{v_{p 0}} a_{e}(v) b_{p 0}(v)}{a_{p 0}(v) a_{e}(v)-\frac{v^{2}}{v_{3}^{2}} b_{p 0}(v)},
$$

with $a_{\star}(v)=1+i \frac{1}{Q_{\star}} \frac{v}{v_{\star}}-\frac{v^{2}}{v_{\star}^{2}}$, where $\star \equiv e, p 0$ and $b_{p 0}(v)=$ $1+i \frac{1}{Q_{p 0}} \frac{\nu}{v_{p 0}}$.

The characteristic frequencies and quality factors inside these expressions are

$$
\begin{aligned}
v_{p 0} & =\frac{1}{2 \pi}\left(L_{J} C_{J}\right)^{-1 / 2}, \quad v_{e}=\frac{1}{2 \pi}\left(L_{e} C_{e}\right)^{-1 / 2}, \\
v_{3} & =\frac{1}{2 \pi}\left(L_{J} C_{e}\right)^{-1 / 2}, \\
Q_{p 0} & =\frac{1}{R_{J}} \sqrt{\frac{L_{J}}{C_{J}}}, \quad Q_{e}=\frac{1}{R_{e}} \sqrt{\frac{L_{e}}{C_{e}}} .
\end{aligned}
$$

The equivalent circuit in Fig. 9 has two modes. A lowfrequency mode determined essentially by the on-chip LC filter connecting the SQUID to the outside world, and a highfrequency mode corresponding to the plasma oscillation of the junction "dressed" by the external circuit:

$$
v_{P}=\frac{1}{2 \pi} \sqrt{\frac{L_{J}^{-1}+L_{e}^{-1}}{C_{J}} .}
$$

Parameters for this equivalent circuit were obtained in the following way. The Josephson junction inductance $L_{J}=$

$595 \mathrm{pH}$ is determined by the critical current extracted from the switching probability measurements. The other five parameters of the equivalent circuit of Fig. 9 were adjusted so as to reproduce at best all the available experimental information. (1) The energy gap measured from the $I-V$ characteristics is $\Delta=194 \mu \mathrm{eV}$. (2) The dressed plasma frequency must be close to $\Delta / 2 h$ in order to explain the position of the sharp threshold observed in the rates $\Gamma_{\text {in }}$ and $\Gamma_{\text {out }}$. This is compatible with what was reported in Ref. [17]. (3) A value of $C_{e}=60 \mathrm{pF}$ is expected from a measurement at very low frequency $(100 \mathrm{kHz})$ on a larger test capacitor fabricated on the same run. (4) The dc subgap current of the $J J$ alone is $I_{J}=4.4 \mathrm{nA}$ at $V_{J}=\Delta / 4 \mathrm{e}$. Imposing the power equality $I_{J} V_{J}=R_{J} I_{0}$ between the $\mathrm{dc}$ injected power and the microwave power absorbed at $\Delta / 2 h$ by the junction's environment, we get $R_{J}=0.3 \Omega$. (5) The low-frequency mode of the environment was measured at $558 \mathrm{MHz}$ in a separate microwave reflectometry experiment [21]. (6) At this resonance frequency, the reflection amplitude $S_{11}$ shows a dip of $-15 \mathrm{~dB}$, from which we determine $R_{e}=0.25 \Omega$.

The two capacitances, $C_{J}$ and $C_{e}$, and the environmental inductance $L_{e}$ were adjusted so as to reproduce the two characteristic frequencies of the circuit. The chosen value $C_{J}=168 \mathrm{fF}$ is a $25 \%$ lower than what is expected from the nominal area of the junction $\left(2.8 \mu \mathrm{m}^{2}\right)$ and the typical specific capacitance for the junctions fabricated usually in our laboratory $\left(75 \mathrm{fF} / \mu \mathrm{m}^{2}\right) . C_{e}=68 \mathrm{pF}$ is a $13 \%$ higher than what is expected from the test low-frequency measurement. Finally, the nominal value $L_{e}=600 \mathrm{pH}$ for the environmental inductance is an $80 \%$ of what is expected from a crude geometrical estimation. With these values we predict a dressed plasma mode frequency of $0.48 \Delta / h$ and a quality factor $Q=116$.

\section{APPENDIX D: RELAXATION DUE TO PHONONS}

The electron-phonon interaction in real space is [26]

$$
\hat{H}_{\mathrm{e}-\mathrm{ph}}=\tilde{\gamma} \int d \mathbf{r} \sum_{\sigma} \Psi_{\sigma}^{\dagger}(\mathbf{r}) \Psi_{\sigma}(\mathbf{r}) \hat{\phi}(\mathbf{r}),
$$

where $\hat{\phi}(\mathbf{r})$ is the phonon field operator:

$$
\hat{\phi}(\mathbf{r})=\sum_{\mathbf{q}} \sqrt{\frac{h v_{\mathbf{q}}}{2 V}}\left(b_{\mathbf{q}} e^{i \mathbf{q r}}+b_{\mathbf{q}}^{\dagger} e^{-i \mathbf{q r}}\right) .
$$

The electron-phonon coupling constant $\tilde{\gamma}$ is $\frac{Z \hbar^{2} \pi^{2}}{m k_{F}} \frac{n_{0}}{B^{\frac{1}{2}}}$, with $n_{0}$ being the atomic density, $B$ is the adiabatic bulk modulus, and $Z$ is the electron valence from the superconductor.

The SC density inside the interaction Hamiltonian takes a form similar to the current operator's in the quasiparticle basis [Eq. (B1)]. However, differently from the coupling with the EM environment, the phonon coupling depends on the geometrical spread of the SC wave functions, a feature characteristic of the coupling of phonons with localized states [24,27].

After eliminating terms linear in $e^{ \pm 2 i k_{F} x}$, which vanish in the spatial integration due to their rapid oscillatory behavior, the matrix element associated to the process $\mid$ Odd, $\sigma\rangle \rightarrow$ $\mid$ Even; $E, e+, \sigma\rangle$ is found to be

$$
\left(C_{(e+)}^{*} C_{A}-D_{(e+)}^{*} D_{A}\right)\left(u_{E} u_{A}-v_{E} v_{A}\right) \frac{e^{-\left(\kappa_{A}+i \kappa_{E}\right) x}}{\sqrt{L \xi_{0}}} .
$$

In the limit $\tau \rightarrow 1$, the squared amplitude of the part of this matrix element that does not depend on $x$, as well as the similar quantity obtained from the matrix element for the process $\mid$ Odd, $\sigma\rangle \rightarrow \mid$ Even; $E, h-, \sigma\rangle$, tend to the expression in Eq. (B6), except for the factor $\left(\hbar e k_{F} / m\right)^{2}$.

It can also be found in the same limit that the analogous $x$-independent quantity for the $|\operatorname{Odd}, \sigma ; E, e-, \bar{\sigma}\rangle \rightarrow \mid$ Even $\rangle$ and $\mid$ Odd, $\sigma ; E, h+, \bar{\sigma}\rangle \rightarrow \mid$ Even $\rangle$ processes is, on the other hand, the same as in Eq. (B5).

The spatial integrals are of the form

$$
\int_{0}^{\infty} d x e^{-\left(\kappa_{A} \pm i \kappa_{E}\right) x} \sin q_{x} x F\left(\mathbf{q}_{\perp}, x\right),
$$

where

$$
F\left(\mathbf{q}_{\perp}, x\right)=\int_{A_{\perp}(x)} d^{2} \mathbf{r}_{\perp} e^{i \mathbf{q}_{\perp} \mathbf{r}_{\perp}}\left|\Psi_{\perp}\left(\mathbf{r}_{\perp}\right)\right|^{2},
$$

$\Psi_{\perp}\left(\mathbf{r}_{\perp}\right)$ being the axial spread of the SC wave functions on the leads, whose geometric details are enclosed in their cross section $A_{\perp}(x)$. 
The momentum transfer $Q$ to or from a phonon taking an active role in these relaxation processes is large compared to the inverse penetration length of the ABS: $Q \gg \kappa_{A}, \kappa_{E}$ in the region $E_{A}(\delta)<\frac{\Delta}{2}$. Following the approximations detailed in Ref. [24] in the theoretical description of the phonon-mediated $\mid$ Even $\left.^{*}\right\rangle \rightarrow \mid$ Even $\rangle$ relaxation, the $F\left(\mathbf{q}_{\perp}, x\right)$ factor introduces a cutoff $\tilde{L}$ in the integral in the $x$ direction,

$$
F\left(\mathbf{q}_{\perp}, x\right) \rightarrow \theta(\tilde{L}-|x|),
$$

and the spatial integration may be easily evaluated in the limit $\tilde{L}^{-1} \gg Q \gg \kappa_{A}, \kappa_{E}$ :

$$
\frac{4 Q \sin Q \tilde{L}}{Q^{2}+\left|\kappa_{A} \pm i \kappa_{E}\right|^{2}} \approx 4 \tilde{L}
$$

Combining the different contributions, the squared amplitude for the process $\mid$ Odd, $\sigma\rangle \rightarrow \mid$ Even; $E, e / h \pm, \sigma\rangle$ mediated by the emission of a phonon is

$$
\left|M_{1}\right|^{2}=8 \frac{h \nu_{Q}}{V} \frac{\tilde{L}^{2} \tilde{\gamma}^{2}}{L \xi_{0}} \sqrt{1-\frac{E_{A}{ }^{2}}{\Delta}} \frac{E^{2}-\Delta^{2}}{E^{2}-E_{A}^{2}} \frac{E-E_{A}}{E} .
$$

The transition rate for such a process is

$$
\frac{2 \pi}{\hbar} V \int \frac{d^{3} \mathbf{Q}}{(2 \pi)^{3}} 4\left|M_{1}\right|^{2} \rho_{S C}(E)\left[1-f_{\mathrm{FD}}\left(E, T_{\mathrm{qp}}\right)\right] f_{\mathrm{BE}}\left(h v_{\mathbf{Q}}, T_{\mathrm{ph}}\right) \delta\left(h v_{\mathbf{Q}}-E+E_{A}\right)
$$

where we have used the same notation as in the main text. We may rewrite the integral over momenta in Eq. (D4) as an integral over energies, with a density of states quadratic in $E-E_{A}$ that appears as a result of this transformation. The resulting total rate is then

$$
\Gamma_{\mathrm{out}}^{(\mathrm{a})}=\frac{2 \pi}{\hbar} \frac{8}{\pi}\left(\frac{\tilde{L}}{\xi_{0}}\right)^{2} \frac{\tilde{\gamma}^{2}}{\pi^{2}}\left(\frac{\Delta}{\hbar c_{s}}\right)^{3} \int_{\Delta}^{\infty} \frac{d E}{\Delta}\left(\frac{E-E_{A}}{\Delta}\right)^{3} g\left(E,-E_{A}\right) f_{\mathrm{BE}}\left(E-E_{A}, T_{\mathrm{ph}}\right)\left[1-f_{\mathrm{FD}}\left(E, T_{\mathrm{qp}}\right)\right] .
$$

Repeating the same process for the process for the form $\mid$ Odd, $\sigma ; E, \eta \bar{\sigma}\rangle \rightarrow \mid$ Even $\rangle$ yields

$$
\Gamma_{\mathrm{out}}^{(\mathrm{b})}=\frac{2 \pi}{\hbar} \frac{8}{\pi}\left(\frac{\tilde{L}}{\xi_{0}}\right)^{2} \frac{\tilde{\gamma}^{2}}{\pi^{2}}\left(\frac{\Delta}{\hbar c_{s}}\right)^{3} \int_{\Delta}^{\infty} \frac{d E}{\Delta}\left(\frac{E+E_{A}}{\Delta}\right)^{3} g\left(E, E_{A}\right)\left[1-f_{\mathrm{BE}}\left(E+E_{A}, T_{\mathrm{ph}}\right)\right] f_{\mathrm{FD}}\left(E, T_{\mathrm{qp}}\right) .
$$

Using the material constants for aluminum, the factor $\frac{2 \pi}{\hbar} \frac{\tilde{\gamma}^{2}}{\pi^{2}}\left(\frac{\Delta}{\hbar c_{s}}\right)^{3}$ is of the order of $10 \mathrm{GHz}$. The phonon rate is reduced because of the relevant thermal factors (which are of the order of $e^{-\beta \Delta} \sim 10^{-5}$ ) and the geometrical factor $\frac{8}{\pi}\left(\frac{\tilde{L}}{\xi_{0}}\right)^{2}$. If this factor is of the order of $10^{-2}$, the phonon-induced relaxation rates are reduced to around $1 \mathrm{kHz}$, which coincide with other estimations in analogous systems [13].

[1] M. H. Devoret and R. J. Schoelkopf, Science 339, 1169 (2013).

[2] J. M. Martinis, M. Ansmann, and J. Aumentado, Phys. Rev. Lett. 103, 097002 (2009).

[3] M. Lenander, H. Wang, Radoslaw C. Bialczak, Erik Lucero, Matteo Mariantoni, M. Neeley, A. D. OConnell, D. Sank, M. Weides, J. Wenner, T. Yamamoto, Y. Yin, J. Zhao, A. N. Cleland, and John M. Martinis, Phys. Rev. B 84, 024501 (2011).

[4] J. Wenner, Yi Yin, Erik Lucero, R. Barends, Yu Chen, B. Chiaro, J. Kelly, M. Lenander, Matteo Mariantoni, A. Megrant, C. Neill, P. J. J. OMalley, D. Sank, A. Vainsencher, H. Wang, T. C. White, A. N. Cleland, and John M. Martinis, Phys. Rev. Lett. 110, 150502 (2013).

[5] G. Catelani, R. J. Schoelkopf, M. H. Devoret, and L. I. Glazman, Phys. Rev. B 84, 064517 (2011).

[6] D. Ristè, C. C. Bultink, M. J. Tiggelman, R. N. Schouten, K. W. Lehnert, and L. DiCarlo, Nat. Comm. 4, 1913 (2013).

[7] Kurtis Lee Geerlings, Ph.D. Thesis, Yale University, 2013.

[8] L. Bretheau, Ç. Ö. Girit, C. Urbina, D. Esteve, and H. Pothier, Phys. Rev. X 3, 041034 (2013).
[9] E. M. Levenson-Falk, F. Kos, R. Vijay, L. Glazman, and I. Siddiqi, arXiv:1310.6996.

[10] M. A. Despósito and A. Levy Yeyati, Phys. Rev. B 64, 140511 (2001).

[11] A. Zazunov, V. S. Shumeiko, E. N. Bratus', J. Lantz, and G. Wendin, Phys. Rev. Lett. 90, 087003 (2003).

[12] N. M. Chtchelkatchev and Yu. V. Nazarov, Phys. Rev. Lett. 90, 226806 (2003).

[13] C. Padurariu and Yu. V. Nazarov, Europhys. Lett. 100, 57006 (2012).

[14] D. Rainis and D. Loss, Phys. Rev. B 85, 174533 (2012).

[15] M. Zgirski, L. Bretheau, Q. Le Masne, H. Pothier, D. Esteve, and C. Urbina, Phys. Rev. Lett. 106, 257003 (2011).

[16] C. W. J. Beenakker, Phys. Rev. Lett. 67, 3836 (1991).

[17] L. Bretheau, C. Girit, H. Pothier, D. Esteve, and C. Urbina, Nature (London) 499, 312 (2013).

[18] Similar processes but for photons from an external driving field were considered in Ref. [8] based on the results of Ref. [19].

[19] F. Kos, S. E. Nigg, and L. I. Glazman, Phys. Rev. B 87, 174521 (2013). 
[20] G. L. Ingold, Yu V. Nazarov, in Single Charge Tunneling, edited by H. Grabert and M. H. Devoret, NATO ASI Series B Vol. 294 (Plenum Press, New York, 1992), pp. 21-107.

[21] L. Bretheau, Ph.D. thesis, Ecole Polytechnique, 2012, available online at http://pastel.archives-ouvertes.fr/pastel-00862029.

[22] See Supplemental Material of Ref. [15].

[23] P. J. de Visser, J. J. A. Baselmans, S. J. C. Yates, P. Diener, A. Endo, and T. M. Klapwijk, Appl. Phys. Lett. 100, 162601 (2012).
[24] A. Zazunov, V. S. Shumeiko, G. Wendin, and E. N. Bratus', Phys. Rev. B 71, 214505 (2005).

[25] G. E. Blonder, M. Tinkham, and T. M. Klapwijk, Phys. Rev. B 25, 4515 (1982).

[26] A. L. Fetter and J. D. Walecka, Quantum Theory of ManyParticle Systems (Dover Publications, Mineola, New York, 2003), p. 397.

[27] D. A. Ivanov and M. V. Feigel'man, JETP Lett. 68, 890 (1998). 